\title{
Aspectos epidemiológicos da leishmaniose visceral no Estado da Bahia, Brasil
}

\author{
Epidemiological aspects of visceral leishmaniasis in the State of Bahia, Brazil \\ Aspectos epidemiológicos de la leishmaniasis visceral en el Estado de Bahía, Brasil
}

Recebido: 21/10/2021 | Revisado: 29/10/2021 | Aceito: 02/11/2021 | Publicado: 05/11/2021

\author{
Isabela Santos Cezar \\ ORCID: https://orcid.org/0000-0003-1100-9978 \\ Centro Universitário Maurício de Nassau, Brasil \\ E-mail: isabelascezar@gmail.com \\ Jéssica Santos Dias de Abreu \\ ORCID: https://orcid.org/0000-0001-5579-5910 \\ Centro Universitário Maurício de Nassau, Brasil \\ E-mail: jessicadiabreu@gmail.com \\ Dahara Keyse Carvalho Silva \\ ORCID: https://orcid.org/0000-0003-4081-1185 \\ Centro Universitário Maurício de Nassau, Brasil \\ E-mail: daharacarvalho@gmail.com \\ Cássio Santana Meira \\ ORCID: https://orcid.org/0000-0002-0113-7864 \\ Centro Universitário Maurício de Nassau, Brasil \\ E-mail: calcio0303@hotmail.com
}

\begin{abstract}
Resumo
O nordeste brasileiro destaca-se por ter uma elevada prevalência de leishmaniose visceral (LV), sendo indicado como a principal região endêmica do Brasil. Tal fato torna de grande valia estudos epidemiológicos na região, visto que ajudam a traçar políticas públicas de saúde mais assertivas para o controle da LV. Nesse contexto, a presente investigação visa delinear o perfil epidemiológico da LV entre os anos de 2007 e 2019 e traçar um perfil de vulnerabilidade frente a doença no estado da Bahia. Trata-se de um estudo seccional, de caráter exploratório, descritivo, quantitativo e retrospectivo. Os dados utilizados relacionados a casos de LV no estado da Bahia são provenientes da base de dados SINAN (Sistema de Informação e Agravos de Notificação). A análise dos dados foi realizada utilizado o programa GraphPad Prism. A Bahia representa o terceiro estado com maior número de casos notificados de LV no nordeste brasileiro entre os anos de 2007 e 2019, com um total de 4.156 casos notificados. Uma análise espacial dessa distribuição demonstra que Juazeiro (146 casos) e Guanambi (125 casos) são os municípios baianos com o maior número de casos notificados no período avaliado. Através de uma análise estratificada dos dados observamos uma maior vulnerabilidade entre os indivíduos do sexo masculino, pardos, com baixo nível de escolaridade e na faixa etária de 1-4 anos. Com o presente trabalho, reforçamos o caráter endêmico da leishmaniose visceral no estado da Bahia e a necessidade de adotar políticas públicas de saúde visando o combate a LV.
\end{abstract}

Palavras-chave: Leishmaniose visceral; Doenças negligenciadas; Epidemiologia.

\begin{abstract}
The northeast of Brazil stands out for having a high prevalence of visceral leishmaniasis (VL), being indicated as the main endemic region of Brazil. This fact makes epidemiological studies in the region of great value, as they help to design more assertive public health policies for the control of VL. In this context, this research aims to outline the epidemiological profile of VL between 2007 and 2019 and outline a profile of vulnerability to the disease in the state of Bahia. This is a sectional, exploratory, descriptive, quantitative and retrospective study. The data used related to VL cases in the state of Bahia come from the SINAN database (Information System and Notifiable Diseases). Data analysis was performed using the GraphPad Prism program. Bahia represents the third state with the highest number of notified VL cases in northeastern Brazil between 2007 and 2019, with a total of 4,156 notified cases. A spatial analysis of this distribution demonstrates that Juazeiro (146 cases) and Guanambi (125 cases) are the municipalities in Bahia with the highest number of reported cases in the period evaluated. Through a stratified analysis of the data, we observed a greater vulnerability among males, browns, with a low level of education and aged 1-4 years. With this work, we reinforce the endemic character of visceral leishmaniasis in the state of Bahia and the need to adopt public health policies aimed at combating VL.
\end{abstract}

Keywords: Visceral leishmaniasis; Neglected diseases; Epidemiology.

\section{Resumen}

El noreste de Brasil se destaca por tener una alta prevalencia de leishmaniasis visceral (LV), siendo indicada como la principal región endémica de Brasil. Este hecho hace que los estudios epidemiológicos en la región sean de gran 
valor, ya que ayudan a diseñar políticas de salud pública más asertivas para el control de la LV. En este contexto, esta investigación tiene como objetivo perfilar el perfil epidemiológico de la LV entre 2007 y 2019 y perfilar un perfil de vulnerabilidad a la enfermedad en el estado de Bahía. Se trata de un estudio seccional, exploratorio, descriptivo, cuantitativo y retrospectivo. Los datos utilizados relacionados con los casos de LV en el estado de Bahía provienen de la base de datos SINAN (Sistema de Información y Enfermedades Notificables). El análisis de datos se realizó utilizando el programa GraphPad Prism. Bahía representa el tercer estado con el mayor número de casos de LV notificados en el noreste de Brasil entre 2007 y 2019, con un total de 4.156 casos notificados. Un análisis espacial de esta distribución demuestra que Juazeiro (146 casos) y Guanambi (125 casos) son los municipios de Bahía con mayor número de casos notificados en el período evaluado. A través de un análisis estratificado de los datos, se observó una mayor vulnerabilidad entre los varones, morenos, con bajo nivel educativo y de 1 a 4 años. Con este trabajo reforzamos el carácter endémico de la leishmaniasis visceral en el estado de Bahía y la necesidad de adoptar políticas de salud pública dirigidas a combatir la LV.

Palabras clave: Leishmaniasis visceral; Enfermedades desatendidas; Epidemiologia.

\section{Introdução}

A leishmaniose visceral (LV) é uma antropozoonose e juntamente com outras doenças parasitárias como malária e doença de Chagas, encontra-se entre as principais doenças negligenciadas do mundo (WHO, 2021a). A OMS estima cerca de 50.000 a 90.000 novos casos de LV por ano e mais de 1 bilhão de pessoas no mundo encontram-se sob risco de desenvolver a infecção (Pérez et al., 2019; DNDI, 2021; WHO, 2021b). Dos novos casos notificados à OMS em 2018, mais de 95\% concentravam-se em 10 países, sendo eles: China, Etiópia, Índia, Iraque, Quênia, Nepal, Somália, Sudão do Sul, Sudão e Brasil, este último representou 97\% dos casos de LV no ano de 2019 nas Américas (Paho, 2020a; WHO, 2021b).

A LV, também conhecida como calazar ou esplenomegalia tropical, é uma doença crônica e sistêmica, que tem como principais sintomas: anemia, febre de longa duração, perda de peso, aumento do fígado e baço (hepatoesplenomegalia), perda ou diminuição da força física (astenia), decréscimo da força muscular e fraqueza (adinamia) (Gomila et al., 2017; Luz et al., 2019). É considerada uma doença com alta letalidade na ausência de tratamento e isso deve-se à disseminação do parasito por diversos órgãos do corpo resultando em alterações hematológicas e infecções bacterianas, as quais contribuem para a ocorrência de um quadro séptico que tende a evoluir para o óbito (Daher et al., 2017; Bispo et al., 2020).

A leishmaniose teve origem em áreas silvestres e rurais, mas, por consequência das mudanças ambientais impulsionadas pela globalização, expandiu-se para o ambiente urbano (BRASIL, 2014, 2016; 2019). O desmatamento em vista da expansão urbana, estimulou a criação de novos bairros e o crescimento populacional, fato que modificou o padrão de transmissão e contribuiu para o surgimento de novos casos em áreas urbanas (Oliveira et al., 2011; Oliveira et al., 2018; Shrestha et al., 2019).

Classicamente, a LV é transmitida por flebotomíneos fêmeas infectadas, popularmente conhecidos como: birigui, anjinho, cangalhinha, tatuquira ou mosquito palha, durante o repasto sanguíneo (Oliveira et al., 2018). No Brasil, os casos de LV estão relacionados a protozoários do gênero Leishmania, sendo a espécie Leishmania chagasi comumente envolvida e o principal vetor é a espécie Lutzomyia longipalpis (Silva et al., 2015; Del Carro et al., 2020; Silva et al., 2021). A região nordeste do Brasil é considerada uma área endêmica de LV nas Américas, devido ao clima favorável à reprodução do inseto vetor (Alves et al., 2016; Rocha et al., 2018; Azevedo et al., 2019; Ribeiro et al., 2021). O nordeste brasileiro, no período de 2007 a 2019 foi a região de maior incidência da LV, com 25.365 casos confirmados e notificados, o que é equivalente a $52,98 \%$ dos casos totais (SINAN, 2021). Dentre os nove estados nordestinos, a Bahia encontra-se na terceira posição, concentrando 4.156 dessas notificações, o que demonstra o caráter endêmico expansivo da doença nesta localidade (SINAN, 2021).

Há 33 anos o nordeste brasileiro destaca-se devido a sua alta taxa de transmissão de LV entre seus municípios, sendo indicado como a principal região endêmica do Brasil (Rocha et al., 2018). Dentre os fatores relacionados ao elevado número de casos, destacam-se: a urbanização descontrolada, elevada taxa de desigualdade social, tendo em vista que, parte da 
população não possui renda suficiente para atender às necessidades suas necessidades básicas, o baixo nível de desenvolvimento regional, e de investimento em saúde e educação (Cardim et al., 2013; Cavalcante \& Vale, 2014; Souza et al., 2020;).

Com base no Guia de Vigilância em Saúde do Ministério da Saúde (2016), a leishmaniose associa-se à pobreza, pois seu risco é aumentado quando há: ausência de medidas preventivas ao indivíduo, condições precárias de saneamento ambiental e de moradia e a falta de qualidade nutricional (BRASIL, 2016). O entendimento acerca da epidemiologia local da doença, habitats de transmissão, o conhecimento da espécie e o contexto social são fatores que contribuem para medidas de prevenção e controle da leishmaniose visceral, a partir da promoção de políticas de saúde pública com o intuito de obter melhores resultados no combate a LV (BRASIL, 2016; 2019; 2021). Desta maneira, além de ações preventivas é estabelecido tratamento medicamentoso, considerando-se questões relevantes relacionadas ao quadro do paciente e a toxicidade da droga, devendo-se utilizar como fármaco de primeira linha antimoniais pentavalentes e como segunda linha, anfotericina B e pentamidina. Estes podem ser usados em combinação ou de maneira isolada (Paho, 2020b; DNDI, 2021; FIOCRUZ, 2021). Nesse contexto, a presente investigação visa delinear o perfil epidemiológico da LV entre os anos de 2007 e 2019 e traçar um perfil de vulnerabilidade frente a doença no estado da Bahia.

\section{Metodologia}

Trata-se de um estudo seccional, de caráter exploratório, descritivo, quantitativo e retrospectivo (Pereira et al., 2018). Os dados utilizados são provenientes da base de dados SINAN (Sistema de Informação e Agravos de Notificação), acessado pelo endereço eletrônico http://www.datasus.gov.br, nos meses de julho e agosto de 2021.

Utilizou-se de forma secundária, dados relacionados a casos de leishmaniose visceral notificados no SINAN no estado da Bahia entre o período de 2007 a 2019. As variáveis analisadas no estudo foram: sexo, faixa etária, raça, município de residência e escolaridade. Os dados extraídos do DATASUS foram utilizados para construção de gráficos, tabelas e mapa através dos programas GraphPad Prism (Graph Pad Software, San Diego, CA, USA), Excel e Tabwin 415 respectivamente. Por se tratar de um banco de domínio público, não foi necessário submeter o projeto ao Comitê de Ética em Pesquisa.

\section{Resultados e Discussão}

Com base em dados obtidos no SINAN (2021), a Bahia representa o terceiro estado com maior número de casos notificados de LV no nordeste brasileiro entre os anos de 2007 e 2019 (SINAN, 2021). Durante esse período foram notificados 4.156 casos de LV na Bahia, sendo este número superado apenas pelos estados do Maranhão e Ceará que apresentaram 6.043 e 6.421 casos notificados, respectivamente. O maior número de casos foi registrado no ano de 2014, quando notificou-se 513 casos de LV no estado, e a média de casos entre os anos de 2007 e 2019 foi de aproximadamente 320 casos por ano (Figura 1). 
Figura 1. Casos confirmados de LV por ano no estado da Bahia (2007-2019).

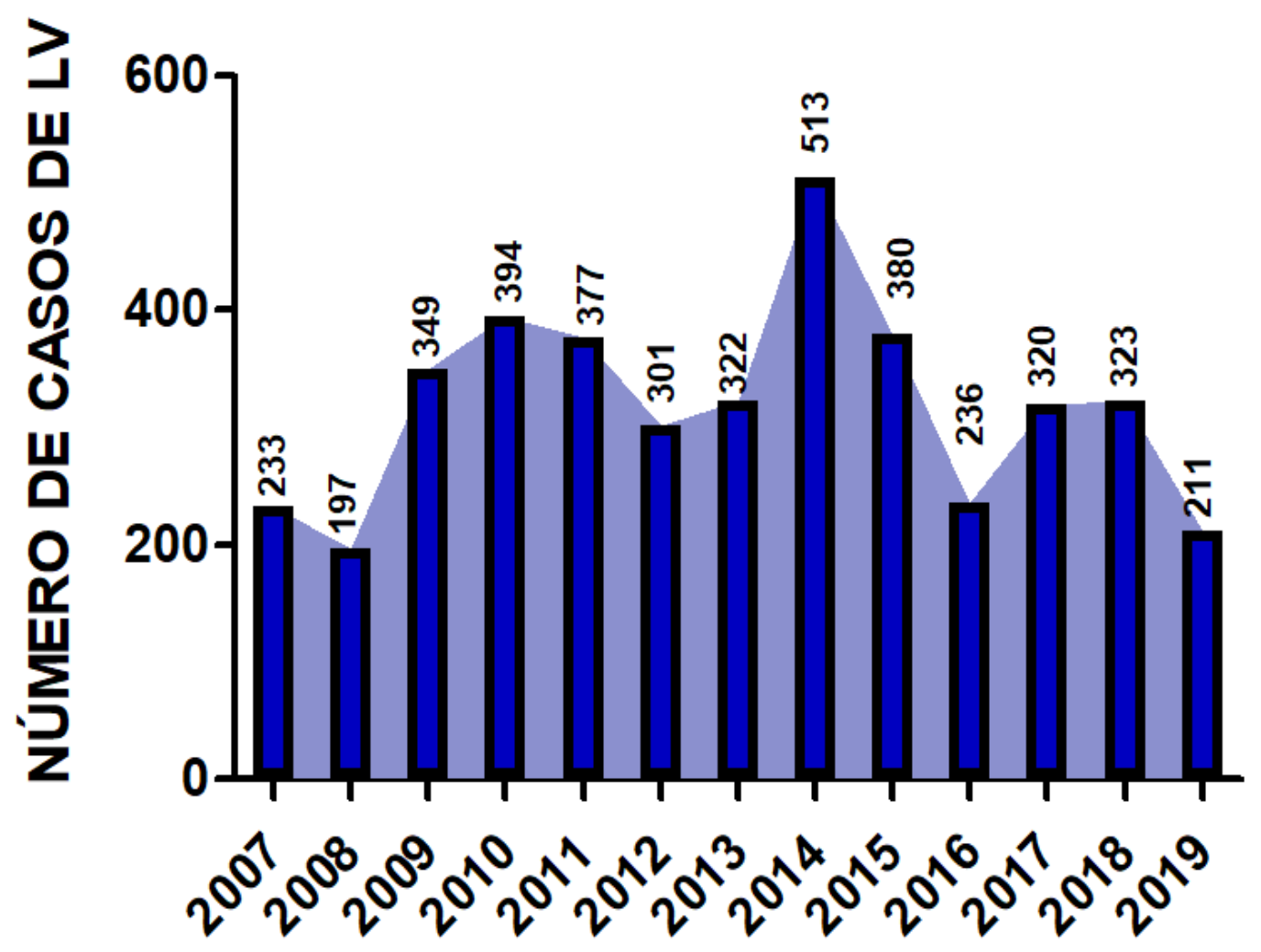

Fonte SINAN-DATASUS (2021).

Uma análise espacial dessa distribuição demonstra que determinados municípios baianos apresentaram uma alta positividade de casos de LV (59 a 146 casos registrados). Como podemos observar na Figura 2, Juazeiro (146 casos), Guanambi (125 casos), Jequié (124 casos), Feira de Santana (116 casos), Salinas da Margarida (103 casos) e Salvador (87 casos) são os municípios baianos com o maior número de casos notificados no período avaliado. Entre estes, Feira de Santana (298) foi a segunda regional de saúde com ascendência de casos no período de 2008 a 2020, de acordo com o Boletim Epidemiológico da Leishmaniose Visceral no Estado da Bahia (2020) (Secretária da Saúde, 2020). 
Figura 2. Casos confirmados de LV por município de residência no estado da Bahia entre os anos de 2007 a 2019.

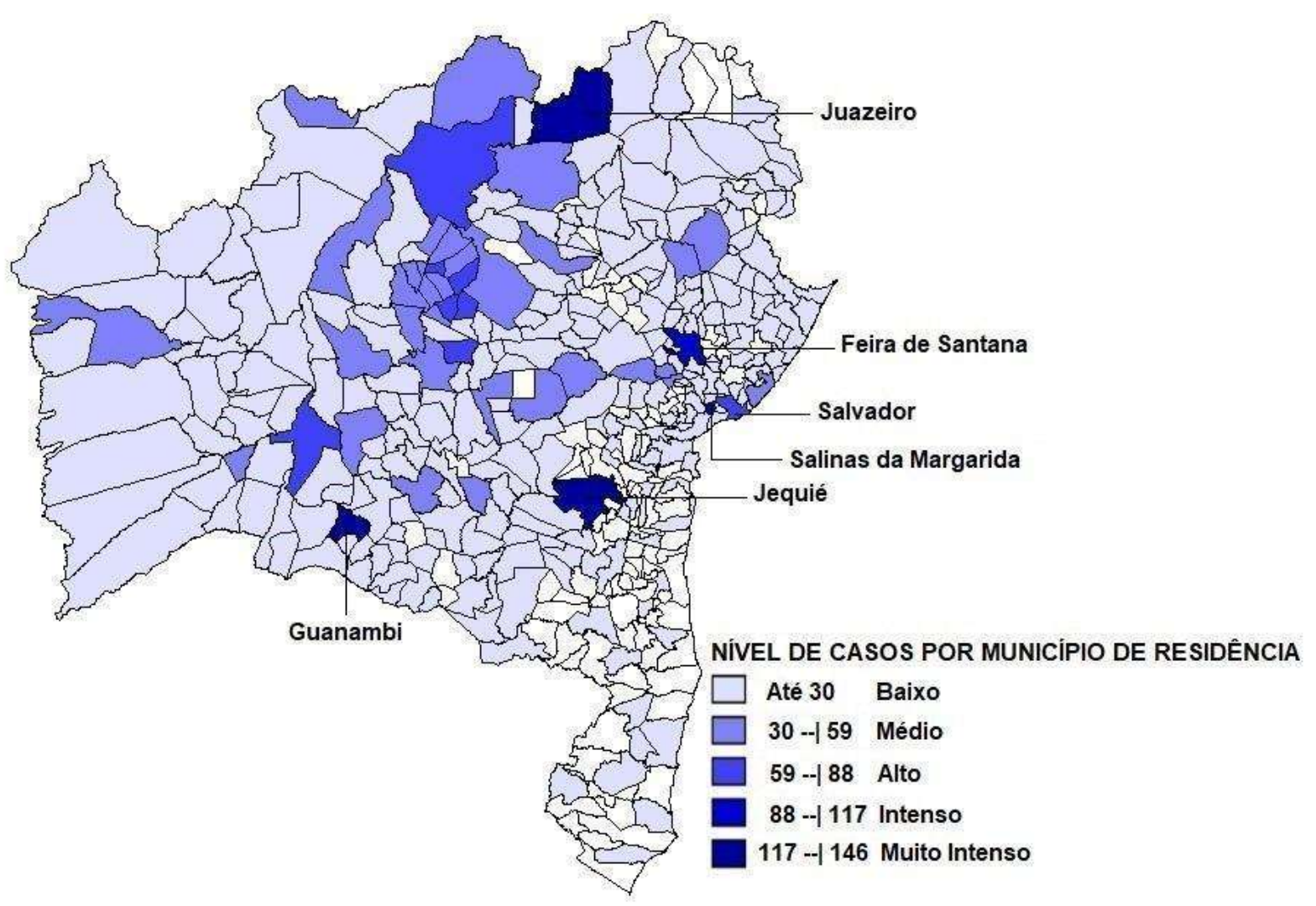

Fonte SINAN-DATASUS (2021).

$\mathrm{Na}$ Bahia, a zona urbana (2.222 casos) representa a principal área endêmica da LV com 51,5\% dos casos confirmados no estado (SINAN,2021). Isso deve-se, principalmente, a expansão urbana que desencadeou desmatamento de áreas de mata e a capacidade adaptativa do vetor (BRASIL, 2021). Essa disseminação não ocorre de maneira aleatória, existe um padrão, compreendido no aumento da temperatura e consequentemente da dispersão vetorial; em condições sociais, entre elas desigualdade socioeconômica, de acesso a serviços de saúde e ensino, favorecendo a propagação do inseto vetor e assim, a transmissão da LV (Góes, 2014; Adegboye et al., 2019; Azevedo et al., 2019).

Através de uma análise estratificada dos dados, é possível notar uma maior vulnerabilidade de indivíduos do sexo masculino frente a LV, tendo em vista que totalizam 2.694 (66\%) dos casos de LV entre os anos de 2007 a 2019, atingindo, em 2014, o maior número de casos confirmados no período avaliado, 308 (61\% dos casos em 2014). Em concordância com os nossos achados, uma maior frequência de casos de LV em homens também foi descrito na população do Ceará, no Brasil como um todo e em Marrocos (Kahime et al., 2017; Reis et al., 2017; Cavalcante et al., 2020). 
Figura 3. Casos notificados de LV por sexo no período de 2007 a 2019 no estado da Bahia.

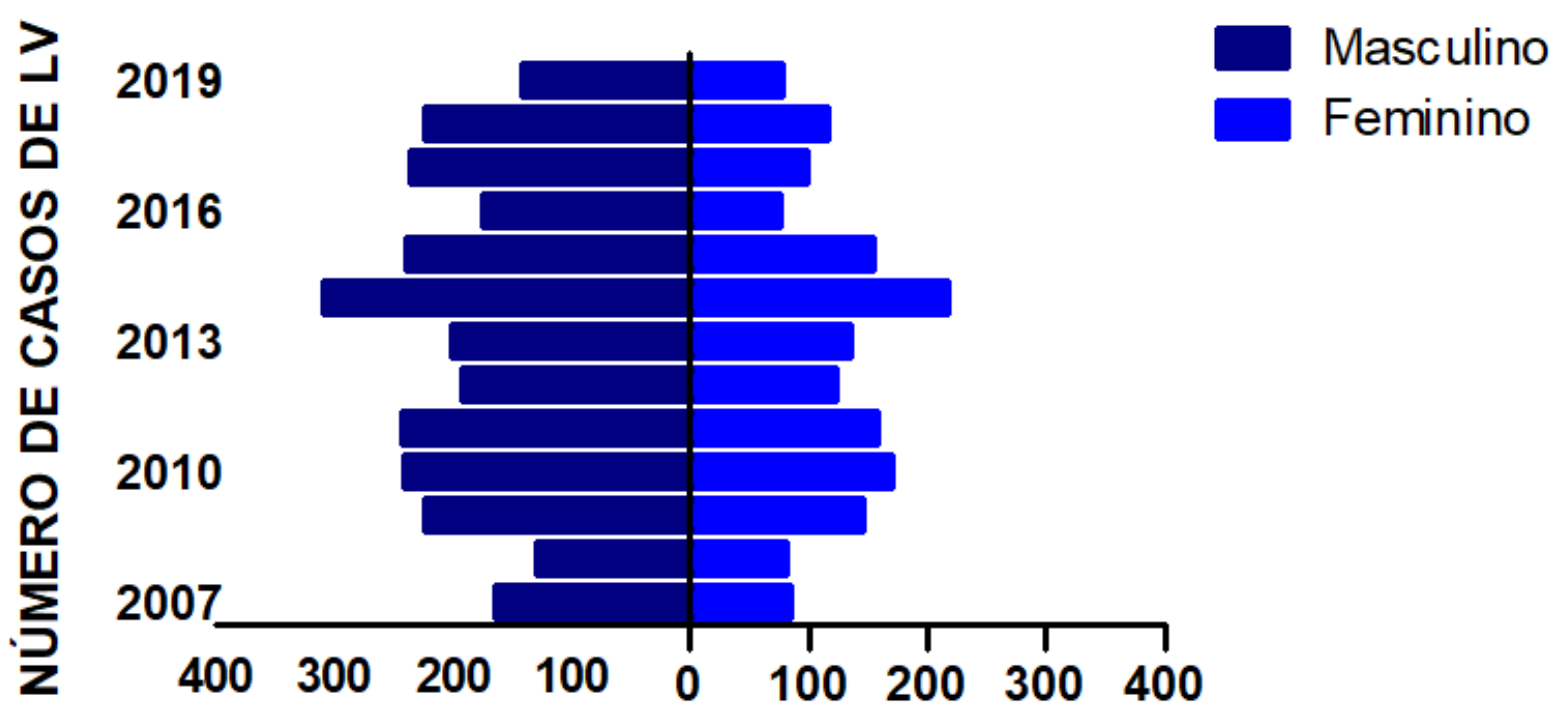

Fonte SINAN-DATASUS (2021).

A suscetibilidade do sexo masculino é correlacionada a fatores hormonais, fisiológicos, ao sistema imunológico e a exposição ao vetor durante atividade laboral (Cardim et al., 2016; Almeida et al., 2020). Cloots e colaboradores (2020), demonstraram através de ensaios in vitro e in vivo, que a testosterona, aumenta a fagocitose de promastigotas (forma infectante) por células fagocíticas e considerou que o hormônio provavelmente tenha influência direta na vulnerabilidade da classe (Cloots et al, 2020).

Em adição, a avaliação da faixa etária demonstrou que crianças entre 1-4 anos, possuem um maior número de notificações positivas da doença na Bahia, sendo contabilizados 1.198 (27,8\%) casos confirmados da LV no estado. O resultado foi semelhante aos estudos de Farias e colaboradores (2019), Rodrigues e colaboradores (2017) e Oliveira e colaboradores (2020) respectivamente realizados no norte de Minas Gerais de 2011 a 2015 onde esta faixa representou 25\% dos casos, em Fortaleza (2009-2013) com destaque no ano de 2010 com 31,5\% dos casos e no estado do Tocantins (20072017) com 1.112 (30\%) casos e de acordo com o Boletim Epidemiológico de doenças tropicais negligenciadas do Ministério da Saúde (2021), crianças menores de cinco anos em 2019 tiveram registros elevados de casos (De Oliveira et al., 2019; Farias et al., 2019; BRASIL, 2021). 
Figura 4. Casos confirmados de LV por faixa etária no estado da Bahia (2007-2019).

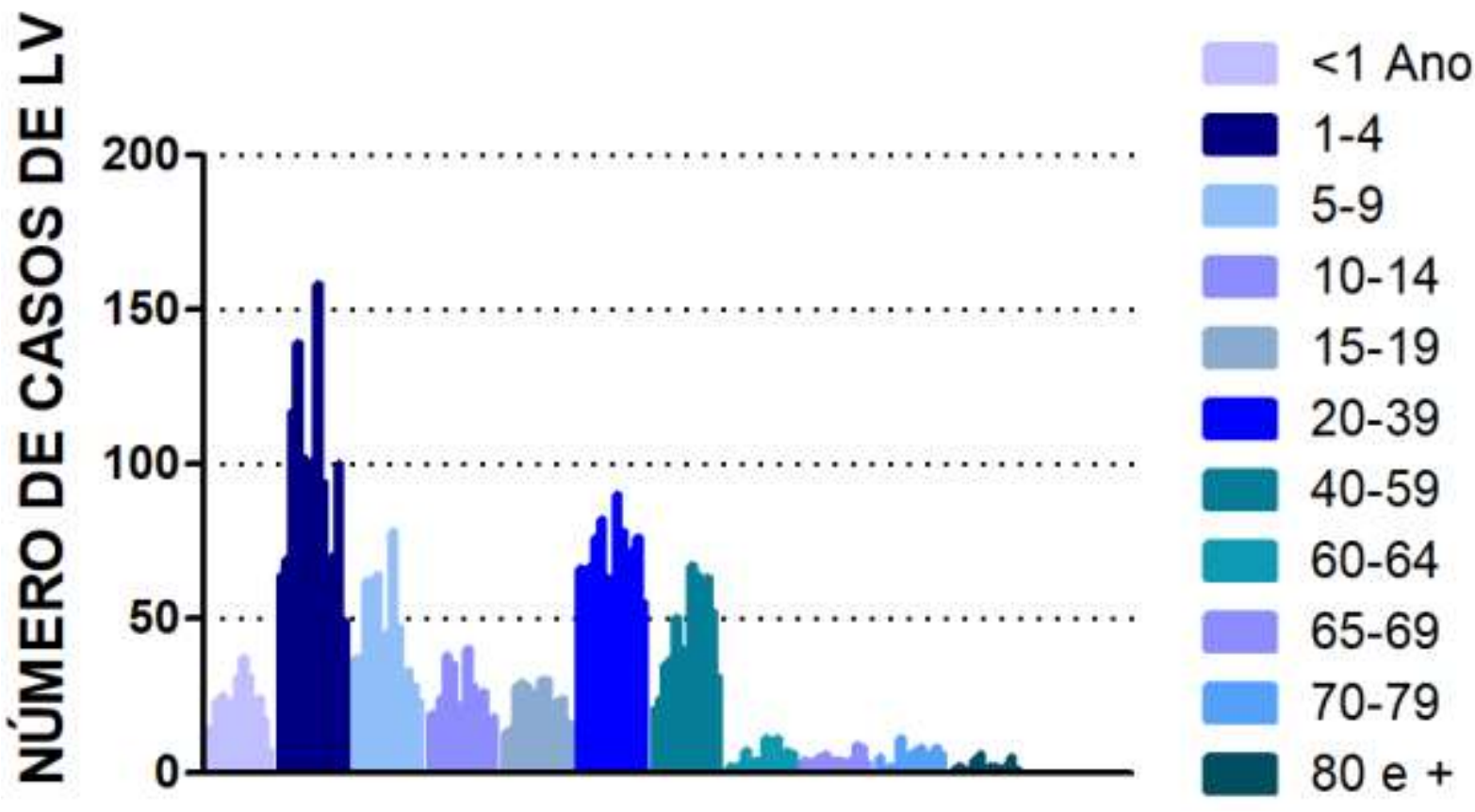

Fonte SINAN-DATASUS (2021).

Outros três grupos demonstraram uma elevada incidência de LV, foram eles: 20-39 anos, com um total de 887 casos confirmados, a faixa de 5 a 9 anos $(571$ casos) e por fim a faixa etária de 40-59 anos, com 563 casos registrados. A Organização Pan-Americana da Saúde - OPAS/OMS (2018), através do Informe Epidemiológico das Américas, apontou as crianças menores de 10 anos, seguidas de indivíduos $\geq 20<50$ anos como grupos mais vulneráveis, resultados este compatível ao encontrado neste estudo (PAHO, 2018). Confere-se ao somatório de casos da LV em crianças 0-4 anos, o perfil imunogenético, a nutrição alimentar, afinidade do mosquito com o grupo, exposição ao ar livre e, consequentemente, ao vetor enquanto brincam e maior proximidade de animais domésticos, que funcionam como reservatórios (Cardim et al, 2016; Amro, 2019; Alebie et al., 2019). Quanto aos segundo e quarto grupos mais acometidos, Almeida e colaboradores (2020), em seu estudo realizado na cidade de Fortaleza (2007-2017), posicionou-se quanto à consequência negativa na economia decorrentes do avanço da LV na faixa etária de 20 a 59 anos. Em adição, Cloots e colaboradores (2020) correlacionou os acometimentos durante a adolescência a possibilidade de terem atingido a puberdade precocemente, obtendo níveis de testosterona elevados que aumenta a atividade fagocítica de formas promastigotas por macrófagos (Almeida et al., 2020; Cloots et al., 2020).

No que tange a raça dos indivíduos acometidos por LV, é notório uma maior prevalência na raça parda 2.727 (63,2\%), seguida pela raça preta $687(15,9 \%)$ e brancas $442(10,3 \%)$ (Figura 5). Porém, esta variável está sujeita a viés tendo em vista que muitos indivíduos pretos se declaram pardos e assim foram registrados em suas respectivas certidões de nascimento. $\mathrm{Na}$ publicação de Sousa e colaboradores (2018) e Luz e colaboradores (2018) a raça parda também foi a que obteve maior frequência de casos reportados (Luz et al., 2018; Sousa et al., 2018). 
Figura 5. Casos notificados de LV por raça (2007-2019) no estado da Bahia.

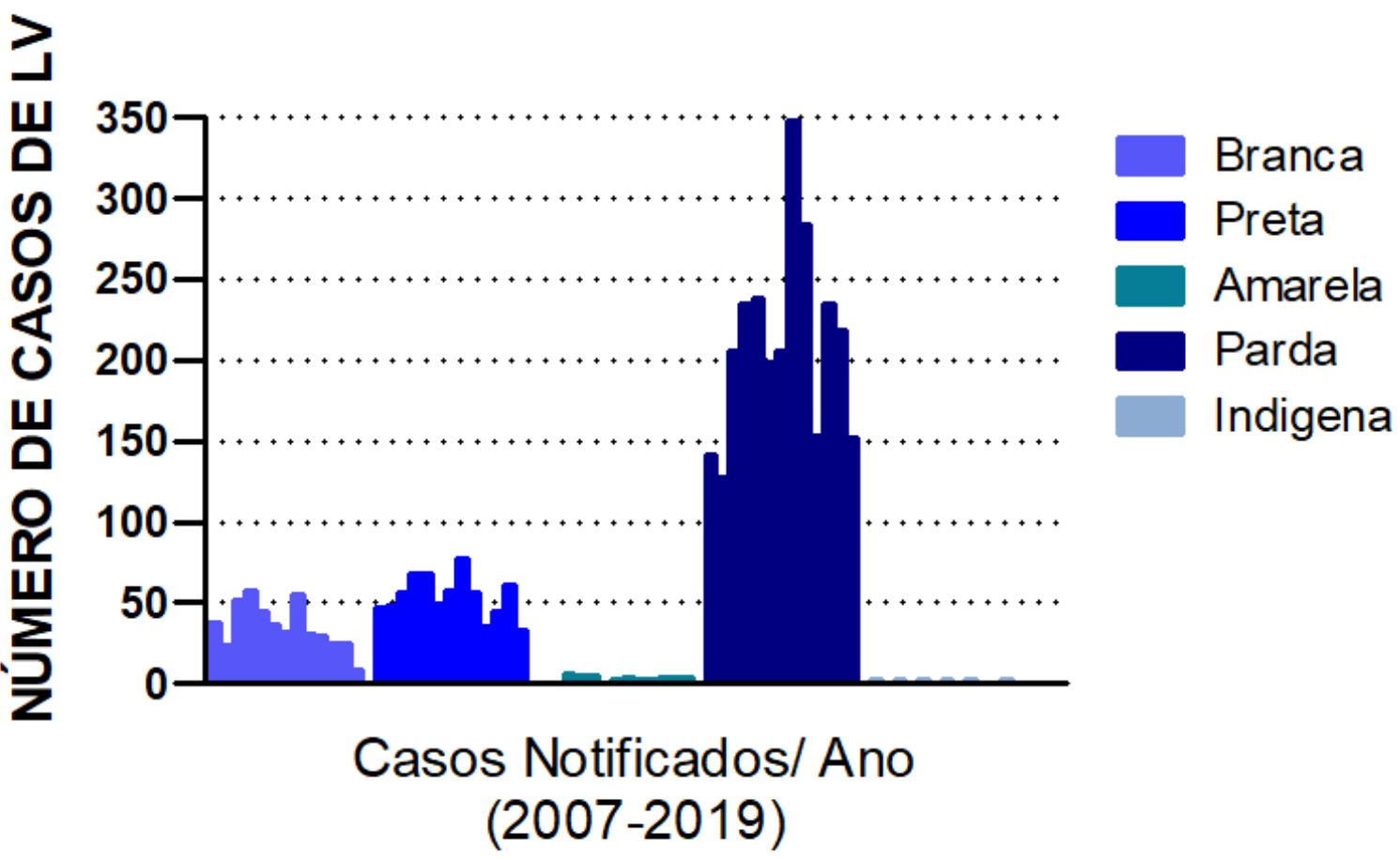

Fonte SINAN-DATASUS (2021).

Apesar da literatura reportar a raça parda com uma maior frequência de casos, existe uma escassez de trabalhos que estabeleçam relações entre a ocorrência de LV e a cor da pele (Sousa et al., 2018). Para Sampaio e colaboradores (2021), mesmo não havendo comprovação quanto a suscetibilidade ou vulnerabilidade da raça face à doença, o risco se eleva em virtude da renda que frequentemente é baixa entre residentes pardos e negros, inviabilizando medidas preventivas e corroborando com o elevado número de casos nesses indivíduos (Sampaio et al., 2021).

Por fim, quanto ao nível de escolaridade no estado da Bahia e o seu reflexo nas notificações da LV, os dados expostos na Figura 6 permitem visualizar um maior número de casos da doença em indivíduos apenas com o ensino fundamental completo, com 1.205 casos notificados $(27,9 \%)$, reforçando que o baixo nível de escolaridade proporciona um conhecimento limitado sobre educação em saúde e déficit socioeconômico, o que contribui com a elevação da prática de exposição aos riscos peridomiciliares (Alves et al., 2016). Em contrapartida, indivíduos com ensino superior no período de análise deste estudo foi o grupo com menor número de casos, apenas 16 indivíduos acometidos, demonstrando o caráter protetor de noções básicas em saúde (Bruhn et al., 2018; Andrade et al., 2020). Essa discrepância entre os níveis de escolaridade foi anteriormente relatada no estudo de Almeida e colaboradores (2020) (Almeida et al., 2020). 
Figura 6. Notificações LV por escolaridade (2007-2019) no estado da Bahia.

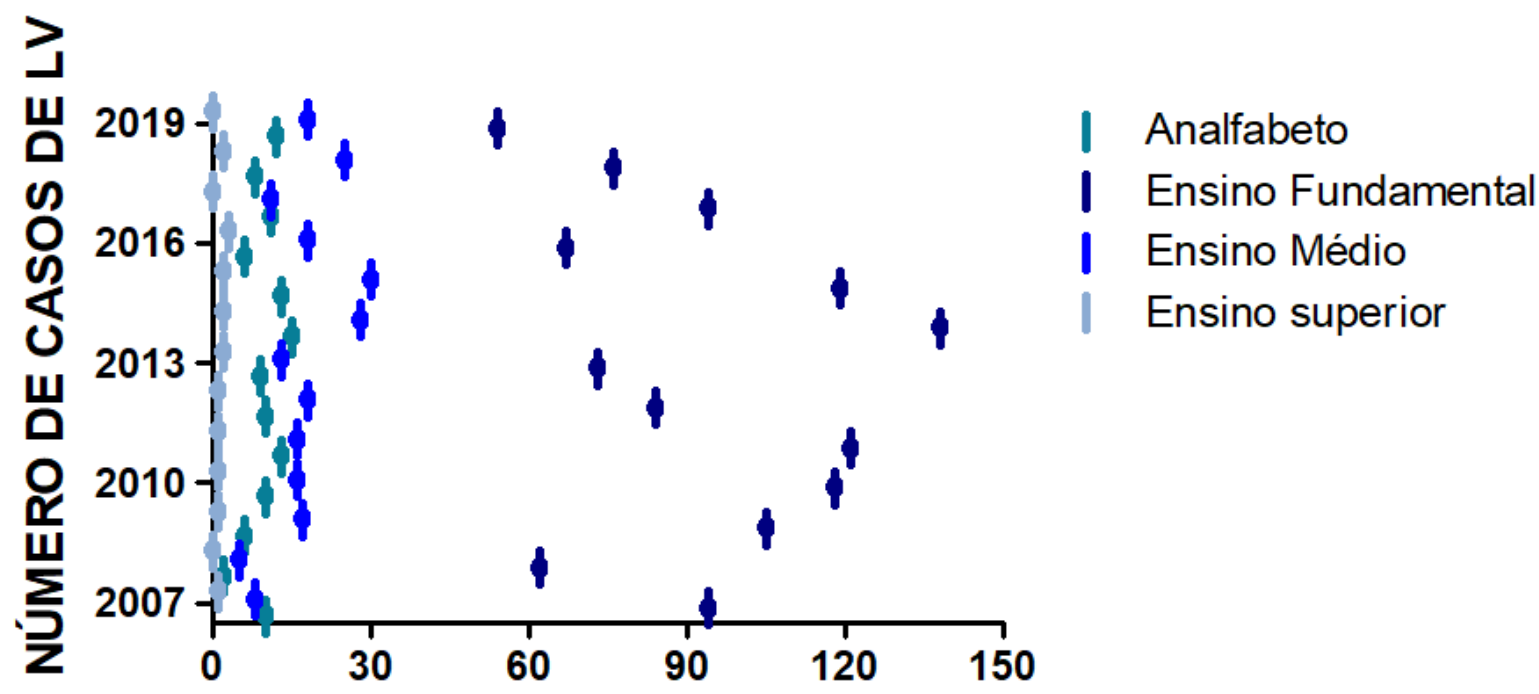

Fonte SINAN-DATASUS (2021).

\section{Considerações Finais}

Com o presente trabalho, reforçamos o caráter endêmico da leishmaniose visceral no estado da Bahia, sendo: Juazeiro, Guanambi, Jequié, Feira de Santana, Salinas da Margarida e Salvador, os municípios baianos com um maior número de casos notificados no período avaliado. Em adição, identificamos indivíduos pertencentes ao sexo masculino, pardos, jovens e com baixo nível de escolaridade, com um maior perfil de vulnerabilidade frente a LV no estado da Bahia. Nesse contexto, a presente investigação reforça a necessidade de adoção de políticas públicas de saúde visando o combate a LV e reitera a importância de trabalhos de natureza epidemiológica, visando uma melhor caracterização da disseminação e a identificação de fatores de riscos da LV no estado da Bahia. Por fim, ressaltamos a importância de trabalhos futuros visando avaliar a eficácia de medidas de combate a disseminação da leishmaniose visceral na Bahia.

\section{Referências}

Adegboye, M. A., Olumoh, J., Saffary, T., Elfaki, F., \& Adegboye, O. (2019). Effects of time-lagged meteorological variables on attributable risk of leishmaniasis in central region of Afghanistan. Rev. Science of The Total Environment, 685, 533-541.

Alebie, G., Worku, A., Yohanmes, S., Urga, B., Hailu, A., \& Tadesse D. (2019) Epidemiology of visceral leishmaniasis in Shebelle Zone of Somali Region, eastern Ethiopia. Parasit Vectors, 12(1), 209.

Almeida C. P., Cavalcante, F. R. A., Moreno, J. O., Florêncio, C. M. G. D., Cavalcante, K. K. S., \& Alencar, H. C. (2020). Leishmaniose visceral: distribuição temporal e espacial em Fortaleza, Ceará, 2007-2017. Epidemiol Serv Saúde, 29(5), 1-11.

Alves, E. B., Costa, C. H. N., Carvalho, F. A. A., Cruz, M. S. P., \& Werneck, G. L. (2016). Risk Profiles for Leishmania infantum Infection in Brazil. Am J Trop Med Hyg, 94(6), 1276-1281.

Amro, A. (2019) Epidemiology and spatiotemporal analysis of visceral leishmaniasis in Palestine from 1990 to 2017. Int J Infect Dis, 90, $206-212$.

Andrade, A. W. F., Souza, C. D. F., \& Carmo, R. F. (2020). Analysis of spatial clustering, time trend, social vulnerability and risk of human visceral leishmaniasis in an endemic area in Brazil: an ecological study. Trans R Soc Trop Med Hyg, 114 (8), 575-584.

Azevedo, T. S., Lorenz, C., \& Chiaravalloti-Neto, F. (2019). Risk mapping of visceral leishmaniasis in Brazil. Rev Soc Bras Med Trop, 52, e20190240.

Bispo, A. J. B., Almeida, M. L. D., Almeida, R. P., Neto, J. B., Brito, A. V. O., \& França, C. M. (2020). Pulmonary involvement in human visceral leishmaniasis: Clinical and tomographic evaluation. PLoS One, 15 (1), e0228176.

Brasil. (2014). Manual de Vigilância e Controle da Leishmaniose Visceral. https://bvsms.saude.gov.br/bvs/publicacoes/manual_vigilancia_controle_leishmaniose_visceral_ledicao.pdf. Acesso, 2021-07-21. 
Brasil. (2016). Guia de Vigilância em Saúde. https://bvsms.saude.gov.br/bvs/publicacoes/guia_vigilancia_saude_led_atual.pdf.

Brasil. (2019). Guia de Vigilância em Saúde. https://bvsms.saude.gov.br/bvs/publicacoes/guia_vigilancia_saude_3ed.pdf.

Brasil. (2021). Boletim Epidemiológico. Doenças tropicais negligenciadas. br/media/pdf/2021/marco/3/boletim_especial_doencas_negligenciadas.pdf.

Bruhn, F. R. P., Morais, M. H. F., Bruhn, N. C. P., Cardoso, D. L., Ferreira, F., \& Rocha, C. M. B. M. (2018). Human visceral leishmaniasis: factors associated with deaths in Belo Horizonte, Minas Gerais state, Brazil from 2006 to 2013. Epidemiologia e Infecção, 146(5), 565-570.

Cardim, M. F. M., Rodas, L. A. C., Dibo, M. R., Guirado, M. M., Oliveira, A. M., \& Neto, F. C. (2013). Introdução e expansão da Leishmaniose visceral americana em humanos no estado de São Paulo. Rev. Saúde Pública, 47 (4), 691-700.

Cardim, M. F. M., Guirado, M. M., Dibo, M. R., \& Chiaravalloti, F. (2016). Leishmaniose visceral no estado de São Paulo, Brasil: análise espacial e espaçotemporal. Rev. Saúde Pública, 50, (48), 1-11.

Cavalcante, I. J. M., \& Vale, M. R. (2014). Epidemiological aspects of visceral leishmaniasis (kala-azar) in Ceará in the period 2007 to 2011 . Rev bras epidemiol, 17(4), 911-924.

Cavalcante, F. R. A., Cavalcante, K.K. S., Florêncio, C. M. G. D., Moreno, J. O., Correia, F. G. S., \& Alencar, C. H. (2020). Human visceral leishmaniasis: epidemiological, temporal and spacial aspects in Northeast Brazil, 2003-2017. Rev Inst Med Trop São Paulo, 62, 1-10 .

Cloots K., Burza, S., Maiaviya, P., Hasker, E., Kansal, S., Mollett, G., Chakravarty, J., Roy, N., Lai, B. K., Rijal, S., Sundar, S., \& Boelaert, M. (2020). Male predominance in reported Visceral Leishmaniasis cases: Nature or nurture? A comparison of population-based with health facility-reported data. PLOS Negl Trop Dis, 14(1), e0007995.

Daher, E. F., Soares, D. S., Filho, S. L. A. P., Meneses, G. C., Freitas, T. V. S., Leite, T. T., \& Junior, G. B. S. (2017). Hyponatremia and risk factors for death in human visceral leishmaniasis: new insights from a cross-sectional study in Brazil. BMC Infect Dis, 17(1), 168.

De Oliveira, M. L., Nascimento, L. S., Carvalho, E. A., \& Machado, F. A. (2019). Análise epidemiológica da leishmaniose visceral no Estado do Tocantins no período de 2007 a 2017. Rev Epidemiol Controle Infecç, 9(4), 316-322.

Del Carro, K. B., Leite, G. R., Filho, A. G. O., Santos, C. B., Pinto, I. S., Fux, B., \& Falqueto, A. (2020). Assessing geographic and climatic variables to predict the potential distribution of the visceral leishmaniasis vector Lutzomyia longipalpis in the state of Espírito Santo, Brazil. PLoS One, 15(9), e0238198.

DNDI (2021). Leishmanioses. https://www.dndial.org/doencas/leishmanioses/.

Dos Reis, L. L., Bailieiro, A. A. S., Fonseca, F. R., \& Gonçalves, M. J. F. (2017) Changes in the epidemiology of visceral leishmaniasis in Brazil from 2001 to 2014. Rev Soc Bras Med Trop, 50 (5), 638-645.

Farias, H. M. T., Gusmão, J. D., Aguiar, R. V., \& Barbosa, S. F. A. (2019). Perfil Epidemiológico da Leishmaniose Visceral Humana nas Regiões de Saúde do Norte de Minas Gerais. Enferm foco, 10 (2), 90-96.

FIOCRUZ. (2021). Leishmaniose. https://portal.fiocruz.br/taxonomia-geral-7-doencas-relacionadas/leishmaniose-O\#: :text $=$ A\%20transmiss $\%$ C3\%A3o\%20acontece\%20quando\%20uma,c\%C3\%A3o\%2C\%20ca valo\%20etc.

Góes, M. A. O., Jeraldo, V. L. S., \& Oliveira, A. S. (2014). Urbanização da leishmaniose visceral: aspectos clínicos e epidemiológicos em Aracaju, Sergipe, Brasil. Rev Bras Med Fam Comunidade, 9(31), 119-126.

Gomila, H. A., Vanzo, C., Garnero, A., Peruzzo, L., \& Badalotti, M. (2017). Visceral leishmaniasis. Pediatric case report. Arch Argent Pediatr, 115(4), 251254.

Kahime, K., Boussaa, S., Nhammi, H., \& Boumezzough, A. (2017). Urbanization of human visceral leishmaniasis in Morocco. Parasite Epidemiol Control, 2(4), 1-6.

Luz, J. G. G., Naves, D. B., Carvalho, A. G., Meira, G. A., Dias, J. V. L., \& Fontes, C. J. F. (2018). Visceral leishmaniasis in a Brazilian endemic area: an overview of occurrence, HIV coinfection and lethality. Rev Inst Med trop S. Paulo, 60. 1-9.

Luz, J. G. G., Carvalho, A. G., Naves, D. B., Dias, J. V. L., \& Fontes, C. J. F. (2019). Where, when, and how the diagnosis of human visceral leishmaniasis is defined: answers from the Brazilian control program. Mem Inst Oswaldo Cruz, 114, e190253.

Oliveira, D. M., Saraiva, E., Ishikawa, E., Sousa, A. A. A., Silva. E., \& Silva, I. M. (2011). Distribution of phlebotomine fauna (Diptera: Psychodidae) across an urban-rural gradient in an area of endemic visceral leishmaniasis in northern Brazil. Mem Inst Oswaldo Cruz, 106(8), 1039-1044.

Oliveira, A.M., López, R. V. M., Dibo, M. R., Rodas, L. A. C., Guirado, M. M., \& Chiaravalloti-Neto, F. (2018). Dispersion of Lutzomyia longipalpis and expansion of visceral leishmaniasis in São Paulo State, Brazil: identification of associated factors through survival analysis. Parasit Vectors, 11(1), 503.

Paho. (2018). Leishmanioses. Informe Américas. https://iris.paho.org/bitstream/handle/10665.2/34857/LeishReport6_por.pdf?sequence=5\#: :text=Em\%202016\%2C\%20foram\%20registrados\%20um,popula $\%$ C3\%A7\% C3\%A3o\%20total\%20do\%20pa\%C3\%ADs\%2C\%20respectivamente.

Paho (2020a). Leishmaniases. Epidemiological Report of the Americas, December 2020. https://www.paho.org/en/documents/leishmaniases-epidemiologicalreport-americas-december-2020.

Paho (2020b). Leishmanioses. Informe Epidemiológico das Américas. https://iris.paho.org/handle/10665.2/53091. 
Research, Society and Development, v. 10, n. 14, e368101422122, 2021

(CC BY 4.0) | ISSN 2525-3409 | DOI: http://dx.doi.org/10.33448/rsd-v10i14.22122

Pereira, A. S., Shitsuka, D. M., Parreira, F. J., \& Shitsuka, R (2018). Metodologia da pesquisa científica. free e-book]. Santa Maria/RS. Ed. UAB/NTE/UFSM.

Pérez-Cabezas, B., Cécilio, P., Gaspar, T. B., Gartner, F., Vasconcellos, R., \& Cordeiro-da-Silva, A. (2019). Understanding Resistance vs. Susceptibility in Visceral Leishmaniasis Using Mouse Models of Leishmania infantum Infection. Front Cell Infect Microbiol, 9:30.

Ribeiro, C. J. N., Santos, A. D., Lima, S. V. M. A., Silva, E. R., Ribeiro, B. V. S., Duque, A. M., Peixoto, M. V. S., Santos, P. L., Oliveira, I. M., Lispcomb, M. W., Araújo, K. C. G. M., \& Moura, T. R. (2021). Space-time risk cluster of visceral leishmaniasis in Brazilian endemic region with high social vulnerability: An ecological time series study. PLoS Negl. Trop. Dis, 15(1), e0009006.

Rocha, M. A. N., Matos-Rocha, T. J., Ribeiro, C. M. B., \& Abreu, S. R. O. (2018). Epidemiological aspects of human and canine visceral leishmaniasis in State of Alagoas, Northeast, Brazil. Braz J Biol, 78 (4), 609-614.

Sampaio, C. K. R. P., Cunha, I. P., Bulgareli, J. V., Guerra, L. M., Gondinho, B. V. C., \& Cortellazzi, K. L. (2021). Leishmaniose visceral na região de SobralCE: perfil epidemiológico dos casos notificados entre os anos de 2015 a 2018. Sanare, 20 (1), 7-16.

Secretaria da Saúde. (2020). Boletim Epidemiológico da Leishmaniose Visceral no Estado da Bahia. http://www.saude.ba.gov.br/wpcontent/uploads/2017/11/boletimEpidimiologicoLeishmanioseVisceral2020.pdf.

Shrestha, M. (2019). Molecular evidence supports the expansion of visceral leishmaniasis towards non-program districts of Nepal. BMC Infect, $19(1)$, 444.

Silva, D.A., Madeira, M. F., \& Figueiredo F. B. (2015). Geographical expansion of canine visceral leishmaniasis in Rio de Janeiro state, Brazil. Rev Inst Med Trop S. Paulo, 57(5), 435- 438.

Silva, T. L., Telleria E. L., \& Csekö Y. M. T. (2021). The gene expression of Leishmania infantum chagasi inside Lutzomyia longipalpis, the main vector of visceral leishmaniasis in Brazil. Mem Inst Oswaldo Cruz, 116, e200571.

SINAN (2021). Leishmaniose Visceral. Brasília: Ministério da Saúde. http://www.portalsinan.saude.gov.br/leishmaniose-visceral.

Sousa, J. M. D. S., Ramalho, W. M., \& De Melo, M. A. (2018). Demographic and clinical characterization of human visceral leishmaniasis in the State of Pernambuco, Brazil between 2006 and 2015. Rev Soc Bras Med Trop, 51 (5), 622-630.

Souza H. P., Oliveira, W. T. G. H., Santos, J. P. C., Toledo, J. P., Ferreira, I. P. S., Esashika, S. N. G. S., \& Lima, T. (2020). Doenças infecciosas e parasitárias no Brasil de 2010 a 2017: aspectos para vigilância em saúde. Rev Panam Salud Publica, 44, e10, 1-7.

WHO (2021a). Control of Neglected Tropical. https://www.who.int/teams/control-of-neglected-tropical-diseases (who.int). Acesso, 2021-07-16.

WHO (2021b). Leishmaniasis. https://www.who.int/news-room/fact-sheets/detail/leishmaniasis. Acesso, 2021-07-16. 